31st October 2018

BARI-TH/00

\title{
On the possibility of measuring the Earth's gravitomagnetic force in a new laboratory experiment
}

\author{
Lorenzo Iorio ${ }^{\dagger}$ \\ †Dipartimento di Fisica dell' Università di Bari, via Amendola 173, 70126, Bari, Italy
}

\begin{abstract}
In this paper we propose, in a preliminary way, a new Earth-based laboratory experiment aimed to the detection of the gravitomagnetic field of the Earth. It consists of the measurement of the difference of the circular frequencies of two rotators moving along identical circular paths, but in opposite directions, on a horizontal friction-free plane in a vacuum chamber placed at South Pole. The accuracy of our knowledge of the Earth's rotation from VLBI and the possibility of measuring the rotators'periods over many revolutions should allow for the feasibility of the proposed experiment.
\end{abstract}




\section{Introduction}

In the weak-field and slow-motion approximation of General Relativity, a test particle in the gravitational field of a slowly rotating body of mass $M$ and angular momentum $\boldsymbol{J}$, assumed to be constant, is acted upon by a non-central acceleration of the form (Ciufolini and Wheeler, 1995; Ruggiero and Tartaglia, 2002)

$$
\boldsymbol{a}_{\mathrm{GM}}=\frac{\boldsymbol{v}}{c} \times \boldsymbol{B}_{\mathrm{g}}
$$

in which $\boldsymbol{v}$ is the velocity of the test particle, $c$ is the speed of light in vacuum and $\boldsymbol{B}_{\mathrm{g}}$ is the gravitomagnetic field given by

$$
\boldsymbol{B}_{\mathrm{g}}=\frac{2 G}{c} \frac{[\boldsymbol{J}-3(\boldsymbol{J} \cdot \hat{\boldsymbol{r}}) \hat{\boldsymbol{r}}]}{r^{3}} .
$$

In it $\hat{\boldsymbol{r}}$ is the unit position vector of the test particle and $G$ is the Newtonian gravitational constant.

For a freely orbiting test particle eq. (II) induces on its orbit the so called Lense-Thirring drag of inertial frames (Lense and Thirring, 1918; Ciufolini and Wheeler, 1995). Such general relativistic spin-orbit effect has been experimentally checked for the first time by analyzing the laser-ranged data to the LAGEOS and LAGEOS II artificial satellites in the gravitational field of the Earth with a claimed accuracy of the order of 20\% (Ciufolini et al., 1998). The use of the proposed LARES satellite would greatly increase the accuracy of such space-based measurement (Ciufolini, 1986; 1998; Iorio et al., 2002a). The famous GP-B mission (Everitt, 2001), whose flight is scheduled for the beginning of 2003, is aimed to the detection, among other things, of a general relativistic spin-spin effect on the orientation of four spaceborne gyroscopes. The claimed accuracy is of the order of $1 \%$.

In regard to the possibility of measuring the terrestrial gravitomagnetic field in an Earthbased laboratory experiment, many experiments have been proposed ${ }^{1}$, but, up to now, none of them has been practically implemented due to relevant practical difficulties. Maybe the most famous of them involves the detection of the gravitomagnetic precession of the swinging plane of a Foucault pendulum at the South Pole (Braginsky et al., 1984). Such proposal would not

\footnotetext{
${ }^{1}$ For a review see chapter 6 of (Ciufolini and Wheeler, 1995) and (Ruggiero and Tartaglia, 2002) and references therein. See also (Braginsky et al., 1977).
} 
be easy to practically be implemented due to many sources of errors recently re-analyzed in (Pascual-Sánchez, 2002). Also the proposal by (Cerdonio et al., 1988) should be mentioned. It is based on an off-line comparison between an astrometric measurement of the Earth's angular velocity and an inertial measurement of the angular velocity of the laboratory. Recently, in (Tartaglia and Ruggiero, 2002) a proposal for detecting the terrestrial gravitomagnetic field by means of electromagnetic waves in a Michelson-Moreley type experiment has been put forth. The effect, in terms of interferometric fringe shift, is really quite small; however, the advances in technology related to the gravitational wave detectors like LIGO and VIRGO might allow for a detection of such effect in future.

Very recently, the influence of a phenomenological gravitational spin-spin coupling on the free fall of mechanical gyroscopes in the terrestrial gravitational field which might violate the equivalence principle (Zhang et al., 2001) has been experimentally investigated (Luo et al., 2002; Zhou et al., 2002) in some laboratory preliminary tests. According to them, it seems that there is no violation of the equivalence principle for extended rotating bodies at the level of $10^{-7}$.

In this paper we intend to present a possible new Earth-based laboratory experiment which exploits, in a certain sense, the concept of the gravitomagnetic clock effect of two counterorbiting test particles along identical circular orbits (Iorio et al., 2002b).

\section{An Earth-based gravitomagnetic clock effect}

According to the gravitational analogue of the Larmor theorem (Mashhoon, 1993), we could obtain eq. (11) by considering an accelerated frame rotating with angular velocity

$$
\Omega_{\mathrm{LT}}=\frac{\boldsymbol{B}_{\mathrm{g}}}{2 c}
$$

Indeed, in it an inertial Coriolis acceleration

$$
\boldsymbol{a}_{\mathrm{Cor}}=2 \boldsymbol{v} \times \Omega_{\mathrm{LT}}
$$

is experienced by the proof mass. It is the same acceleration which, among other things, induces the Lense-Thirring precession of the swinging plane of the Foucault pendulum in the 
experiment proposed by Braginsky and coworkers (Braginsky et al., 1984) by means of the component of $\boldsymbol{\Omega}_{\mathrm{LT}}$ along the local vertical direction.

Let us choose an horizontal plane at, say, South Pole ${ }^{2}$ : here the Earth's angular velocity vector $\boldsymbol{\omega}_{\oplus}$ and $\boldsymbol{\Omega}_{\mathrm{LT}}$ are perpendicular to it and have opposite directions. Let us choose as unit vector for the $z$ axis the unit vector $\hat{\Omega}_{\mathrm{LT}}$, so that

$$
\begin{aligned}
\boldsymbol{\Omega}_{\mathrm{LT}} & =\frac{2 G J}{c^{2} R_{\mathrm{p}}^{3}} \hat{\boldsymbol{z}} \\
\boldsymbol{\omega}_{\oplus} & =-\omega_{\oplus} \hat{\boldsymbol{z}} \\
\boldsymbol{g} & =-g \hat{\boldsymbol{z}}
\end{aligned}
$$

where $\boldsymbol{J}$ is the proper angular momentum of the Earth $^{3}, R_{\mathrm{p}}$ is the Earth polar radius and $\boldsymbol{g}$ is the gravitoelectric acceleration to which, at the poles, the centrifugal acceleration does not contribute.

A particle which moves with velocity $\boldsymbol{v}$ in such polar horizontal plane is acted upon by the Coriolis inertial force induced by the noninertiality of the terrestrial reference frame ${ }^{4}$ and also by the gravitational force of eq. (4). Such forces have the same line of action and opposite directions: in an horizontal plane at South Pole the resultant acceleration is $2 v \tilde{\Omega} \equiv 2 v\left(\Omega_{\mathrm{LT}}-\omega_{\oplus}\right)$ and it lies in the plane orthogonally to $\boldsymbol{v}$.

Let us consider an experimental apparatus consisting of a friction-free horizontal plane placed in a vacuum chamber. Upon such a desk a small tungsten mass $m$, tied to a sapphire fiber of length $l$, tension $\boldsymbol{T}$ and fixed at the other extremity, is put in a circular uniform motion. Indeed, the forces which act on $m$ are the tension of the wire, the Coriolis inertial force and the

\footnotetext{
${ }^{2}$ Of course, it is easier to prepare an experimental setup in the Antarctic continent rather than in the Arctic floe. Thanks to the small size of the proposed apparatus, it should not be too difficult to find an Antarctic region free enough from seismic noise and other geological disturbances. Last but not least, several scientific stations already exist in the Antarctic continent.

${ }^{3}$ In fact, while $\boldsymbol{J}$ remains constant, the Earth's angular velocity vector $\boldsymbol{\omega}_{\oplus}$ does not (Bertotti and Farinella, 1990). Indeed, among other things, it moves around $\boldsymbol{J}$ with an approximate period of 14 months (the Chandler wobble) due to the oblateness of the Earth (motion with respect to the terrestrial reference frame). Moreover, there are also the secular precession of the equinoxes induced by the external lunisolar torque on the equatorial bulge with a period of 26,000 years and other faster variations (motion with respect to the celestial reference frame). The angular velocity of the Earth $\omega_{\oplus}$ suffers a secular deceleration due to the lunar torque so that the length of day increases in a year by about $2 \times 10^{-5} \mathrm{~s}$. In addition, we have other changes in $\omega_{\oplus}$ over shorter time scales due to moving masses within and on the Earth, in particular the oceans and the atmosphere. However, over the characteristic time scales of the experiment all such variations of $\boldsymbol{\omega}_{\oplus}$ can be neglected.

${ }^{4}$ Of course, the centrifugal force is absent at the Earth's Poles.
} 
Lense-Thirring gravitational force which are all directed radially; the weight force $\boldsymbol{W}=m \boldsymbol{g}$ is balanced by the the normal reaction $\boldsymbol{N}$ of the plane and there are neither the atmospheric drag nor the friction of the plane. Let us assume the counterclockwise rotation as positive direction of motion for $m$. At the equilibrium the equation of motion is

$$
m \omega_{+}^{2} l=T-2 m \omega_{+} l \tilde{\Omega},
$$

where $\omega_{+}$is the angular velocity of the mass $m$ when it rotates counterclockwise and $l$ is the radius of the circle described by $m$. If the Earth did not rotate the angular velocity of the particle would be

$$
\omega_{0}=\sqrt{\frac{T}{m l}}
$$

For, say, $m=100 \mathrm{~g}, T=m g=9.798 \times 10^{4}$ dyne and $l=100 \mathrm{~cm} \omega_{0}=3.13 \mathrm{rad} \mathrm{s}^{-1}, P_{0}=2$ s. The gravitomagnetic and the Coriolis forces slightly change such circular frequency. Since $\omega_{0}>>\tilde{\Omega}$, from eq. (8) it follows for both the counterclockwise and clockwise directions of rotation

$$
\omega_{ \pm}=\omega_{0} \mp \tilde{\Omega}
$$

so that we could adopt as observable

$$
\Delta \omega \equiv \omega_{-}-\omega_{+}=2 \tilde{\Omega} \equiv 2\left(\Omega_{\mathrm{LT}}-\omega_{\oplus}\right)
$$

Of course, the physical properties of the sapphire fiber and of the tungsten mass should not change from a set of rotations in a direction to another set of rotations in the opposite direction, so to allow an exact cancellation of $\omega_{0}$ in eq. (11).

Since on the Earth's surface at the poles $\Omega_{\mathrm{LT}}=3.4 \times 10^{-14} \mathrm{rad} \mathrm{s}^{-1}$, would the experimental sensitivity of the sketched apparatus allow to measure such so tiny effect? If we measure the frequency shift $\Delta \omega$ from the rotational periods of the mass $m$ we have

$$
\delta \Omega_{\mathrm{LT}}=\frac{\delta(\Delta \omega)^{\exp }+\delta \omega_{\oplus}}{2}
$$

with

$$
\delta(\Delta \omega)^{\exp }=\delta \omega_{-}^{\exp }+\delta \omega_{+}^{\exp }=2 \pi\left[\left(\frac{\delta P_{-}}{P_{-}^{2}}\right)^{\exp }+\left(\frac{\delta P_{+}}{P_{+}^{2}}\right)^{\exp }\right]
$$


The Earth's angular velocity $\omega_{\oplus}$ is very well known in a kinematically, dynamically independent way from the Very Long Baseline Interferometry (VLBI) technique with an accuracy of the order of ${ }^{5} \delta \omega_{\oplus} \sim 10^{-18} \mathrm{rad} \mathrm{s}^{-1}$. In fact, $\omega_{\oplus}$ is not exactly uniform and experiences rather irregular changes which are monitored in terms of Length-Of-Day (LOD) by the Bureau Internationale des Poids et Mesures-Time Section (BIMP) on a continuous basis ${ }^{6}$. Such changes are of the order of $\Delta \omega_{\oplus} \sim 0.25$ milliarcseconds per year $\left(\operatorname{mas~yr}^{-1}\right)=3.8 \times 10^{-17} \mathrm{rad} \mathrm{s}^{-1}$, so that they are negligible. A possible source of error might come from our uncertainty in the position of the proposed polar set-up with respect to the Earth's crust, i.e. from the polar motion of the instantaneous axis of rotation of the Earth $\hat{\boldsymbol{\omega}}_{\oplus}$ in terms of the small angles $x$ and $y$. It turns out that this phenomenon has three components: a free oscillation with a (measured) period of 435 days (Chandler Wobble) and an amplitude of less than 1 arcsecond (asec), an annual oscillation forced by the seasonal displacement of the air and water masses of the order of $10^{-1}$ asecs and an irregular drift of the order of some asecs. There are also some diurnal and semi-diurnal tidally induced oscillations with an amplitude less than 1 mas. As a consequence, the position of the pole is unknown at a level of some meters. The small size of the apparatus should overcome such problem. Moreover, it can be easily seen that the impact of such offsets of $\hat{\boldsymbol{\omega}}_{\oplus}$ on the Coriolis force is $2 v \omega_{\oplus} \cos \delta$ with $\delta$ of the order of some asecs or less, so that it is negligible.

In regard to the experimental measurement of the periods $P_{ \pm}$, it should be possible to strongly constrain eq. (13) by choosing suitably the parameters of the apparatus so to increase the periods and/or by measuring them after many revolutions.

\section{Discussion and Conclusions}

In this paper we have proposed a new experiment for measuring the gravitomagnetic LenseThirring effect in an Earth-based laboratory set-up.

The key point consists of the measurement of the difference of the rotational frequencies

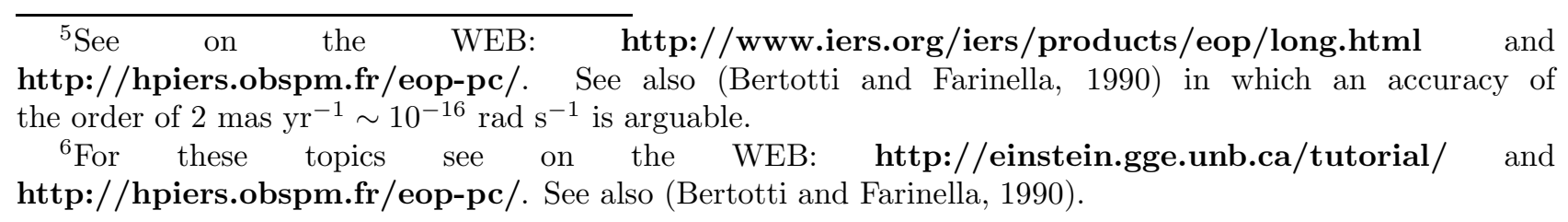


of two test bodies which rotates uniformly along the same circular paths, but in opposite directions, in an horizontal, friction-free plane in a vacuum chamber at the South Pole. The value of the Earth's daily rotation rate has to be subtracted from such quantity, but the great accuracy in our knowledge of it from VLBI would allow to single out the tiny relativistic effect. Over many revolutions it should be possible to experimentally measure the difference of the rotational frequencies from the periods to a sufficiently high level of accuracy to allow for an extraction of the investigated gravitomagnetic effect.

Of course, many practical difficulties would make the proposed measurement very hard to be implemented. For example, it turns out that the the friction force of the plane should be

less than $2 \times 10^{-9}$ dyne. Moreover, in order to reach the quoted accuracy in measuring $\omega_{\oplus}$ with VLBI several years of continuous observation would be required.

\section{References}

[Bertotti and Farinella, 1990] Bertotti B., and P. Farinella, Physics of the Earth and the Solar System, Kluwer Academic Publishers, Dordrecht, 1990.

[Braginsky et al., 1977] Braginsky, V.B., C.M. Caves, and K.S. Thorne, Laboratory experiments to test relativistic gravity, Phys. Rev. D, 15(8), 2047-2068, 1977.

[Braginsky et al., 1984] Braginsky, V.B., A.G. Polnarev, and K.S. Thorne, Foucault Pendulum at the South Pole: Proposal For an Experiment to Detect the Earth's General Relativistic Gravitomagnetic Field, Phys. Rev. Lett., 53(9), 863-866, 1984.

[Cerdonio et al., 1988] Cerdonio, M., G. Prodi, and A. Vitale, Dragging of Inertial Frames by the Rotating Earth: Proposal and Feasibility for a Ground-Based Detection, Gen. Rel. and Grav., 20(1), 83-87, 1988.

[Ciufolini, 1986] Ciufolini, I., Measurement of Lense-Thirring drag on high-altitude, laser ranged artificial satellites, Phys. Rev. Lett., 56(4), 278-281, 1986.

[Ciufolini, 1998] Ciufolini, I., The Concept of the LARES experiment, in LARES Phase-A Study, pp. 16-33, Rome, 1998. 
[Ciufolini et al., 1998] Ciufolini, I., E. Pavlis, F. Chieppa, E. Fernandes-Vieira, and J. PérezMercader, Test of General Relativity and Measurement of the Lense-Thirring Effect with Two Earth Satellites, Science, 279, 2100-2103, 1998.

[Ciufolini and Wheeler, 1995] Ciufolini, I., and J.A. Wheeler, Gravitation and Inertia, Princeton University Press, New York, 1995.

[Everitt et al., 2001] Everitt, C. W. F., and other members of the Gravity Probe B team, Gravity Probe B: Countdown to Launch, in: Lämmerzahl, C., C.W.F. Everitt, and F.W. Hehl (Eds.), Gyros, Clocks, Interferometers...:Testing Relativistic Gravity in Space, Lecture Note in Physics 562, 507 pp., Springer Verlag, Berlin, 2001.

[Iorio et al., 2002a] Iorio, L. D.M. Lucchesi, and I. Ciufolini, The LARES mission revisited: an alternative scenario, Class. and Quantum Grav., 19(16), 4311-4325, 2002a.

[Iorio et al., 2002b] Iorio, L., H.I.M. Lichtenegger, and B. Mashhoon, An alternative derivation of the gravitomagnetic clock effect, Class. and Quantum Grav., 19(1), 39-49, 2002b.

[Lense and Thirring, 1918] Lense, J., and H. Thirring, Über den Einfluss der Eigenrotation der Zentralkörper auf die Bewegung der Planeten und Monde nach der Einsteinschen Gravitationstheorie, Phys. Z., 19, 156-163, 1918, translated by Mashhoon, B., F. W. Hehl, and D. S. Theiss, On the Gravitational Effects of Rotating Masses: The Thirring-Lense Papers, Gen. Rel. Grav., 16, 711-750, 1984.

[Luo et al., 2002] Luo, J., Y.X. Nie, Y.Z. Zhang, and Z.B. Zhou, Null result for violation of the equivalence principle with free-fall rotating gyroscopes, Phys. Rev. D, 65(4), 042005, 2002.

[Mashhoon, 1993] B. Mashhoon, On the Gravitational Analogue of Larmor Theorem, Phys. Lett. A, 173, 347-354, 1993.

[Pascual-Sánchez, 2002] Pascual-Sánchez, J.F., TELEPENSOUTH project: Measurement of the Earth gravitomagnetic field in a terrestrial laboratory, to appear in: FernandezJambrina, L., and L.M. Gonzalez-Romero (Eds.), Relativistic Astrophysics, Lecture Notes in Physics, Springer Verlag, Berlin, preprint gr-qc/0207122, 2002. 
[Ruggiero and Tartaglia, 2002] Ruggiero, M.L., and A. Tartaglia, Gravitomagnetic effects, to be published in Il Nuovo Cimento, preprint gr-qc/0207065, 2002.

[Tartaglia and Ruggiero, 2002] Tartaglia, A., and M.L. Ruggiero, Angular Momentum Effects in Michelson-Morley Type Experiments, Gen. Rel. and Grav., 34(9), 1371-1382, 2002.

[Zhang et al., 2001] Zhang, Y.Z., J. Luo, and Y.X. Nie, Gravitational Effects of Rotating Bodies, Mod. Phys. Lett. A, 16(12), 789-794, 2001.

[Zhou et al., 2002] Zhou, Z.B., J. Luo, Q. Yan, Z.G. Wu, Y.Z. Zhang, and Y.X. Nie, New upper limit from terrestrial equivalence principle test for extended rotating bodies, Phys. Rev. D, 66(2), 022002, 2002. 